\title{
Lipopeptides as Dimerization Inhibitors of HIV-1 Protease
}

\author{
Hans J . Schramm ${ }^{1, *}$, Eve de Rosny ${ }^{2}$, \\ Michèle Reboud-Ravaux ${ }^{2}$, J ochen Büttner ${ }^{3}$, \\ Andrea Dick ${ }^{3}$ and Wolfgang Schramm ${ }^{3}$ \\ ${ }^{1}$ Max-Planck-Institut für Biochemie, \\ Am Klopferspitz 18a, D-82152 Martinsried, Germany \\ e-mail: schramm@biochem.mpg.de \\ ${ }^{2}$ Institut J . Monod, Universités P aris 6 and 7, 2, \\ place J ussieu, F-75251 Paris, France \\ ${ }^{3}$ Universitätsklinikum (Innenstadt) der Universität, \\ Ziemssenstr. 1, D-80336 München, Germany \\ * Corresponding author
}

\begin{abstract}
In AIDS therapy, attempts have been made to inhibit the virus-encoded enzymes, e.g. HIV-1 protease, using active site-directed inhibitors. This approach is questionable, however, due to virus mutations and the high toxicity of the drugs. An alternative method to inhibit the dimeric HIV protease is the targeting of the interface region of the protease subunits in order to prevent subunit dimerization and enzyme activity. This approach should be less prone to inactivation by $\mathrm{mu}$ tation. A list of improved 'dimerization inhibitors' of HIV-1 protease is presented. The main structural features are a short 'interface' peptide segment, including non-natural amino acids, and an aliphatic $\mathrm{N}$-terminal blocking group. The high inhibitory power of some of the lipopeptides [e.g. palmitoyl-Tyr-Glu-Leu-OH, palmitoyl-Tyr-Glu-(L-thyronine)-OH, palmitoyl-Tyr-Glu(L-biphenyl-alanine)-OH] with low nanomolar $\mathrm{K}_{\mathrm{i}}$ values in the enzyme test suggests that mimetics with good bio-availability can be derived for AIDS therapy. Key words: AIDS / Dimerization inhibitors / HIV-1 protease / Lipopeptides / Modified peptides.
\end{abstract}

Inhibitors of the viral key enzyme HIV-1 protease (PR) prevent viral infectivity and are considered to provide the most effective treatment of AIDS, especially in the form of a combination therapy with inhibitors of HIV reverse transcriptase. However, the toxicity of the drugs and the rapid appearance of mutant viruses with proteases resistant to active site inhibitors have led to doubt about the present therapy regimens (for a recent review, see Flexner, 1998). There is common consent, therefore, that alternative approaches are urgently needed.

While enzymes are usually inactivated by blocking of the active site, the structure of the dimeric PR allows an alternative inhibitory mechanism. Since the active site is formed by both half-enzymes - which are connected by a $\beta$-sheet formed of the four intercalated terminal amino acid segments at the 'interface' (Wlodawer and Vondrasek, 1998), - reagents that interfere with formation or stability of the functional PR dimer can abolish activity by 'dimerization inhibition'. This mode of inhibition was observed for peptides with the $\mathrm{N}$ - or C-terminal amino acid sequences (Zhang et al., 1991, Schramm et al., 1991), and led to the identification of improved inhibitory peptides (Babé et al., 1992, Franciskovich et al., 1993, Uhliková et al., 1996, Zutshi et al., 1997, Fan et al., 1998; Bouras et al., 1999) and to the formulation of a 'consensus sequence' lle-Ser-Tyr-Glu-Leu (with possible conservative amino acid exchanges) for dimerization inhibitors (Schramm et al., 1996). This structure emphasizes the $\beta$ sheet characteristics of the inhibitory peptides, the value of two negative charges in positions 98 (Glu orAsp) and 99 (free $\mathrm{C}$-terminus), the hydrophobic side chain in position 99 and, especially, the anchor residue Tyr in position 97 (using the sequence numbering of PR). It should be noted that most of these important structural features reside in the short sequence 97 to 99 , a tripeptide core structure. The replacement of some of the amino acids by non-natural amino acids further improves the inhibitory power and supplies more precise knowledge about the structural properties of good dimerization inhibitors (C aflisch et al., in preparation). It has also been shown (Schramm et al., 1996) that a large N-terminal aliphatic blocking group such as the palmitoyl residue - not only improves the inhibitory potency of such peptides but also acts as an interface directing group.

In this study, the N-terminal blocking group was not varied at first, in order to limit the parameters. Instead, the optimal length of the peptide moiety was investigated using short sequences with variable length and composition, including some non-natural amino acids commercially available for peptide synthesis. The data of Table 1 further confirm that the attached large, lipid moiety improves binding and directs peptides to the dimerization interface. The main result is that in the enzyme assay even short 3 mer peptides combined with the palmitoyl residue were excellent PR inhibitors. The best inhibitors so far are the tripeptides palmitoyl-Tyr-Glu-Leu-OH, palmitoyl-Tyr-Glu(biphenylalanine)-OH and palmitoyl-Tyr-Glu-(L-thyronine)$\mathrm{OH}$. In these structures, two of the amino acids (95 and 96) of the 'consensus' pentamer peptide could be omitted but the main attachment groups (see above) of the core trimer are retained. In addition, the large 'bumpy' side chain of biphenylalanine may not fit well into the corresponding binding sub-site of the dimer active site, and also in this way direct the inhibitor to the monomer interface. The contrary seems to be true for palmitoyl-Tyr-Glu-Tyr-OH, an 
Table 1 Inhibition Constants of Peptides for HIV-1 Protease.

\begin{tabular}{|c|c|c|c|c|}
\hline Peptide & $\begin{array}{l}\mathrm{IC}_{50}(\mathrm{~nm}) \\
(\mathrm{PR} \text { conc/nm) }\end{array}$ & $\mathrm{K}_{\mathrm{i}}^{\text {dim }}(\mathrm{nm})$ & $\mathrm{K}_{\mathrm{i}}^{\mathrm{com}}(\mathrm{nM})$ & Purity (\%) \\
\hline $\mathrm{H}$-Tyr-Glu-Leu-OH ${ }^{b}$ & 75000 & 2400 & - & $>96$ \\
\hline $\mathrm{H}$-Tyr-Asp-Leu-OH ${ }^{b}$ & 9000 & n.d. & n.d. & $>96$ \\
\hline Ac-Tyr-Glu-Leu-OHb & 34000 & n.d. & n.d. & 99 \\
\hline Ac-Tyr-Glu-Trp-OH ${ }^{b}$ & 21000 & n.d. & n.d. & 99 \\
\hline Octanoyl-Tyr-Glu-Leu-OH $\%$ & n.d. & 1000 & 1000 & 95 \\
\hline 4-Phenylbutyroyl-Tyr-Glu-Leu-OH\% & n.d. & 5000 & $(11000)$ & 95 \\
\hline Pam-Tyr-Glu-Leu-OH ${ }^{\&}$ & $150^{\mathrm{a}}$ & $11^{\mathrm{a}}$ & - & $>70$ \\
\hline Pam-Tyr-Glu-Tyr-OH & n.d. & - & 2000 & 95 \\
\hline Pam-Tyr-Glu-(pBzF)-OH & $50(50)$ & 120 & - & $>90$ \\
\hline Pam-Tyr-Glu-(Bip)-OH & $25(50)$ & 10 & - & $>80$ \\
\hline Pam-Tyr-Glu-(2Nal)-OH & $500(20)$ & n.d. & n.d. & $>60$ \\
\hline Pam-(pBzF)-Glu-Leu-OH ${ }^{\varepsilon}$ & ca. $100(10)$ & n.d. & n.d. & $>60$ \\
\hline Pam-(2Nal)-Glu-Leu-OH & 200 & n.d. & n.d. & $>60$ \\
\hline Pam-Tyr-Glu-(L-thyronine)-OH) & ca. 50 (4) & 5 & n.d. & $>95$ \\
\hline $\mathrm{H}$-Ser-Tyr-Glu-Leu-OH & n.d. & 1300 & - & 99 \\
\hline H-Ser-Tyr-Glu-Try-OH ${ }^{b}$ & n.d. & 300 & - & 99 \\
\hline Ac-Ser-Tyr-Glu-Leu-OH ${ }^{b}$ & n.d. & 300 & - & 99 \\
\hline Pam-Ser-Tyr-Glu-Leu-OH ${ }^{\AA}$ & ca. 300 & ca. 40 & - & $>75$ \\
\hline Pam-Ser-(pBzF)-Glu-Leu-OH & $400(10)$ & n.d. & n.d. & $>70$ \\
\hline Pam-Ser-(2Nal)-Asp-Leu-OH & 200 & n.d. & n.d. & $>85$ \\
\hline Pam-Ser-(2Nal)-Glu-(2Nal)-OH & $230(50)$ & n.d. & n.d. & $>90$ \\
\hline H-Phe-Ser-Tyr-Glu-Leu-OH & $700^{a}$ & - & $600^{\mathrm{a}}$ & $>95$ \\
\hline Ac-Ile-Ser-Tyr-Glu-Leu-OH & n.d. & - & $600^{a}$ & $>95$ \\
\hline Ac-lle-Ser-(pBzF)-Glu-Leu-OH & n.d. & - & $600^{\mathrm{a}}$ & $>95$ \\
\hline Ac-Ile-Ser-(2Nal)-Glu-Leu-OH & 300 & - & 260 & $>80$ \\
\hline Ac-Phe-Ser-Tyr-Glu-Leu-OH & $900^{\mathrm{a}}$ & - & $900^{\mathrm{a}}$ & $>95$ \\
\hline Ac-Phe-Ser-(pBzF)-Glu-Leu-OH & $300,600^{a}$ & - & $400^{\mathrm{a}}$ & n.d. \\
\hline Pam-Phe-Ser-Tyr-Glu-Leu-OH & ca. 100 & n.d. & n.d. & $>65$ \\
\hline Pam-Phe-Ser-(pBzF)-Glu-Leu-OH & 100 & n.d. & n.d. & n.d. \\
\hline Palmitic acid & no inhibition & - & - & 99 \\
\hline Ethyl palmitate & no inhibition & - & - & 99 \\
\hline
\end{tabular}

The inhibition constants of HIV-1 protease inhibitors are shown together with the purity of the investigated peptide samples.

Sources: HIV-1 protease was produced by bacterial expression in E. coli (Schramm et al., 1996; Billich et al., 1990). Peptides were purchased from Neosystem Lab., Strasbourg, France, or from Biogenes, Berlin, Germany. The purity of the peptides was not always high, the HPLC data of the peptides never showed major additional peaks. The nonapeptide substrate was synthesized at the Max-Planck-Institut für Biochemie, Martinsried. Other organic compounds were purchased from Sigma-Aldrich, Steinheim, Germany, and Paris, France, respectively. The fluorogenic substrate was purchased from Bachem Biochemie SARL, Voisins-le-Bretonneux, France.

Methods: Most $\mathrm{K}_{\mathrm{i}}$ and $\mathrm{IC}_{50}$ values were determined using the nonapeptide substrate H-Lys-Ala-Arg-Val-Nle · (p-nitro-Phe)-Glu-Ala-NleNH2 (Richards et al., 1990), at pH 4.7, 4 mM EDTA, 5 mm DTT, 0.1 m sodium acetate, $0.1 \mathrm{M} \mathrm{NaCl}$ (Schramm et al., 1996). The samples were added in DMSO with a total concentration of $3 \%$ DMSO (v/v).

${ }^{a}$ The original method of Zhang et al., 1991, with the fluorogenic substrate 4-(4-dimethylamino-phenyl-azo)benzoyl-4-aminobutyryl-SerGln-Asn-Tyr-Pro-Ile-Val-4-aminobutyryl- $N^{5}$-[(5-sulfonaphthyl)-2-aminoethyl]-Gln-Arg (Matayoshi et al., 1990) was also used. The fluorescence measurements were performed in $0.1 \mathrm{~m}$ sodiumacetate, $1 \mathrm{mM}$ EDTA, $0.1 \mathrm{M} \mathrm{NaClatpH} 4.7$, and a total concentration of $3 \%$ DMSO (v/v) using a obin Yvon spectrofluorometer. For the determination of the $\mathrm{IC}_{50}$ values, the enzymatic reaction was initiated by HIV- 1 PR (final concentration: $15 \mathrm{~nm}$ or $30 \mathrm{~nm}$ ) to a buffer containing $5.2 \mu \mathrm{m}$ substrate and at least 5 different inhibitor concentrations. The increase in fluorescence at $490 \mathrm{~nm}\left(\lambda_{\mathrm{exc}}=340 \mathrm{~nm}\right)$ was monitored over a period of $5 \mathrm{~min}$ at $30^{\circ} \mathrm{C}$. For the kinetic analysis according to Zhang et al., 1991, of Pam-Tyr-Glu-Leu-OH the substrate concentration was $5.2 \mu \mathrm{M}$, the enzyme concentrations ranged from 9.4 to $47.2 \mathrm{~nm}$, the inhibitor concentrations from 0.28 to $0.56 \mu \mathrm{M}$. For Ac-Phe-Ser-Tyr-Glu-Leu-OH the enzyme concentrations ranged from 9.4 to $37.7 \mathrm{~nm}$, the inhibitor concentration was $2.8 \mu \mathrm{M}$. 2-Naphthylalanine peptides could not be analysed with fluorogenic substrate because of fluorescence interference. With some of the peptides the $K_{i}$ determination was difficult, or not possible at all, because of instable absorbance readings, possibly due to the formation of aggregates or micelles.

${ }^{b}$ Data already published (Schramm et al., 1996). $\mathrm{K}_{i}^{\text {dim }}$, inhibition constant with dimerization inhibition mode according to Zhang et al., 1991. $\mathrm{K}_{\mathrm{i}}^{\mathrm{com}}$, inhibition constant with active site binding.

Abbreviations: pBzF, para-benzoylphenyl-alanine; 2Nal, 2-naphthyl-alanine; Bip, p-biphenyl-alanine; dim =dimerization inhibition; com =competitive inhibition; n.d., not determined. \&, solubility problems; \%, mixed type inhibition (Schramm et al., 1996).

active site inhibitor, where the exchange of leucine to tyrosine in position 99 showed no improvement with respect to the mode of dimerization inhibition.
Subsequently, in some experiments the crucial palmitoyl group was altered. Size reduction of the $\mathrm{N}$-terminal blocking group was expected to reduce binding and 
specificity. Indeed, an exchange of the C-16 palmitoyl to the C-8 octanoyl blocking group reduced binding power and produced a partly active site-directed inhibitor. The flexible fatty acid chain probably contributes by covering one of the two hydrophobic cavities below the $\beta$-sheet (one on either side of the anchor residue Tyr97), which are partly solvent-exposed in the monomers. The site next to the peptide C-terminus is flanked by amino acids Prol, Ile3, Val11, Leu24, Cys67 and His69. The other - rather open - 'sub-site' can be reached by the palmitoyl group from the peptide $\mathrm{N}$-terminus and is formed mainly by the residues Leu5, Thr26 and Leu97. These subsites are not identical with the active site subsites S $1, S 2$ and S3, which are only partly retained in the PR monomer. The residual parts cannot easily be reached from the interface. The Nterminal octanoyl group is not large enough for covering enough surface to prevent subunit dimerization completely. Similarly, the 4-phenyl-butyroyl blocking group is not able to replace the palmitoyl group in this binding subsite. In both cases, a 'mixed mode' (Schramm et al., 1996) is obtained. With some justification, one can describe the palmitoyl peptides, therefore, as 'bifunctional' inhibitors, binding to the $\beta$-sheet as well as to the hydrophobic subsites. The enlargement of the side-chain in position 99 (using tryptophan, biphenyl-derivatives and other groups instead of leucine) may also have such a specificity and binding improving effect, and is being studied further.

The representation of the kinetic constants of Pam-TyrGlu-Leu in the plots proposed by Zhang et al., 1991, shows the parallel line pattern proposed for dimerization inhibition (s. Figure 1) in contrast to the converging line pattern in the case of active site inhibition. So far, kinetic equilibrium constants of highly potent dimerization inhibitors of PR have not be reported. Now, a clear kinetic pattern characteristic for dimerization inhibition has been found for active inhibitors with $\mathrm{K}_{\mathrm{i}}^{\text {dim }}$ constants in the low nanomolar region. However, because of the limited purity of some of the analyzed peptides and some technical difficulties (due to mi-

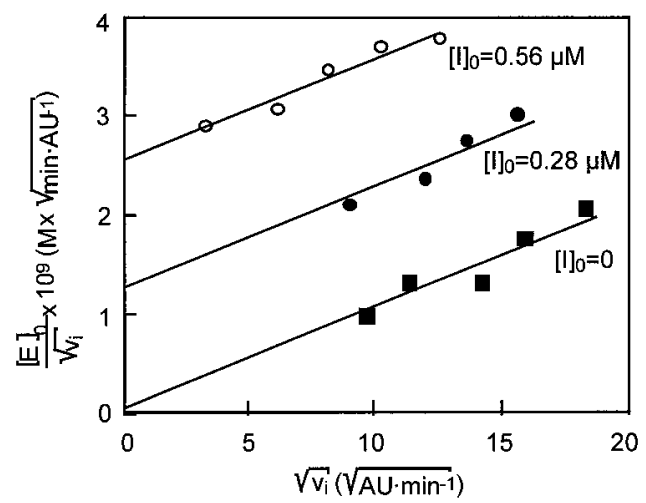

Fig. 1 Zhang-Poorman-Plot for the Inhibition of PR by Palmitoyl-Tyr-Glu-Leu.

$[E]_{0} / \sqrt{ } v_{i}$ is plotted versus $\sqrt{ } v_{i}$ (Zhang et al., 1991) for the hydrolysis of fluorogenic substrate (see legend of Table 1 ) by HIV-1 protease in the presence of palmitoyl-Tyr-Glu-Leu-OH. $\mathrm{K}_{\mathrm{i}}^{\text {dim }}$ is derived from the y intercept. Buffer: $0.1 \mathrm{~m}$ sodium acetate, $0.1 \mathrm{~m}$ sodium chloride, $1 \mathrm{~mm}$ EDTA, $\mathrm{pH} 4.7, \mathrm{~K}_{\mathrm{i}}=11 \mathrm{~nm}$. celle formation?), the obtained kinetic constants must be considered as preliminary values. As previously reported (Schramm et al., 1996), the $K_{i}$ values determined by different methods (with fluorogenic versus chromogenic substrates) are identical with regard to the level of accuracy. Values obtained by the different enzymatic tests can be compared, therefore. In spite of the above limitations, the main conclusion - the high inhibitory power of short lipopeptides against PR and their mode of action - is not in doubt.

To find the smallest still sufficiently active peptide part appears useful, since passive membrane permeability is increased for small peptides with a border value of about $M_{r}=650$, although it is not clear how hydrophobic residues influence this value. The low $M_{r}$ value of only 662 of palmitoyl-Tyr-Glu-Leu suggests that derivatives (e.g. esters, as 'pro-drugs', modified peptides etc.) may be small and hydrophobic enough for passive cell entry. Furthermore, receptor targeting could be attempted by attaching receptor binding groups to the $\mathrm{N}$-terminus of peptides (or modified peptides) replacing the palmitoyl group.

Enzyme/inhibitor complexes are usually studied by Xray or NMR structure determinations. If the inhibitor is bound to the interface region of a monomeric PR subunit, these techniques are not applicable, since the necessary high concentrations of PR shift the equilibrium toward the dimer. For that reason, this type of inhibition may be found only in dilute PR solutions, and only under near neutral $\mathrm{pH}$ and low ionic strength conditions (in contrast to the low pH and the high salt concentrations often used). Since in cells the ionic strength is only about $0.15 \mathrm{M}$ and the $\mathrm{pH}$ neutral, this mechanism of PR inhibition may well occur. Even some 'established' active site inhibitors (by X-ray techniques, i.e. under high salt conditions), as well as several regulatory peptides derived from viral proteins ( $p 6^{*}$, vif), may also act as dimerization inhibitors in vivo. There are some ill-understood synergy effects of active site in hibitors (e.g. Klimkait et al., 1996) that may be explained in this way.

The principle of dimerization inhibition (or 'interface targeting') has been applied already to several protein assemblies (for a recent review see Zutshi et al., 1998). It has not been extensively investigated in those cases where $\beta$-structures are involved in dimerization. Since the resemblance of some other $\beta$-sheet structures to the PR interface is evident, one can assume that lipopeptides also bind to such other $\beta$-structures, e.g. by insertion into parallel $\beta$-sheet strands or addition to aggregates. Such structurally related targets may be amyloid deposits (e.g. of Alzheimer or prion diseases) or the insertion sites of serpins. It is possible, therefore, that modified lipopeptides may be us eful for analysis (staining of amyloid deposits) or treatment (dissolving of $\beta$-aggregates, inhibition of regulatory serpins), especially if the hydrophobic group is modelled complementary to the relevant binding site. Vice versa, serpin 'insertion peptides' may act on PR. A first result supporting this notion is the finding that insertion peptides of $\alpha 1$ proteinase inhibitor (Schulze et al., 1992) can act as 
PR inhibitors with an inhibitory power in the low micromolar range (unpublished).

The high enzymatic activity in connection with the small size and cheap synthesis - developing countries cannot afford the high costs of the present therapy regimens identifies the lipopeptidic inhibitors as interesting prototypes for the development of alternative PR inhibitors.

\section{Acknowledgements}

We thank Frank Siedler, MPI B iochemie, for the preparation of the chromogenic substrate and Traudl Wenger, MPI Biochemie, for the preparation of HIV protease and for some tests. We also thank Rene Loic and Bernard Badet, ICSN-CNRS, Gif-sur-Yvette, for a high purity sample of Pam-Tyr-Glu-Leu-OH. This work was supported by grants from the Deutsche Forschungsgemeinschaft (Schr 95/13) and the BIOMED-2 program of the European Commission (PL 950824). The results have been presented in part at the Congress 'Drug Therapy in AIDS Infection', Glasgow, 8-12 November, 1998 (AIDS 12, suppl. 4, P239).

\section{Note added in Proof}

The $\mathrm{IC}_{50}$ value for Pam-Tyr-Glu-Leu-OH has been confirmed using a newly synthesized product $>99 \%$ pure, chromogenic substrate).

\section{References}

Babé, L.M., Rosé, J ., and Craik, C.S. (1992). Synthetic 'interface' peptides alter dimeric assembly of the HIV-1 and 2 proteases. Protein Science 1, 1244 - 1253.

Billich, A., Hammerschmidt, F., and Winkler, G. (1990). Purification, assay and kinetic features of HIV-1 proteinase. Biol. Chem. Hoppe-Seyler 371, 265 - 271.

Bouras, A., Bogetto, N., Benatalah, Z., de Rosny, E., Sicsic, S., and Reboud-Ravaux, M. (1999). J . Med. Chem. 42, 957 - 962.

Flexner, Ch. (1998). HIV-Protease Inhibitors. New Engl. J . Med. $338,1281-1292$.

Fan, X., Flentke, G.R., and Rich, D.H. (1998). Inhibition of HIV-1 protease by a subunit of Didemnaketal A. J . Amer. Chem. Soc. $120,8893-8894$.

Franciskovich, J., Houseman, K., Mueller, R., and Chmielewski, J .
(1993). A systematic evaluation of the inhibition of HIV-1 protease by its $\mathrm{C}$ - and $\mathrm{N}$-terminal peptides. Bioorg. \& Medicin. Chem. Lett. 3, 765- 768.

Klimkait, T., "The Ciba HIV-P rotease Team” (1996). CGP 61755, a potential combination partner for Saquinavir: A synergistic HIV-1 protease inhibitor with high antiviral potency. Antiviral. Res. 30, A33 (51), Abstract.

Matayoshi, E.D., Wang, G.T., Krafft, G.A., and Erickson, J . (1990). Novel fluorogenic substrates for assaying retroviral proteases by resonance energy transfer. Science 247, $954-958$.

Richards, A.D., Phylip, L.H., Farmerie, W.G., Scarborough, P.E., Alvarez, A., Dunn, B.M., Hirel, P.-H., Konvalinka, J ., Strop, P., Pavlickova, L., Kostka, V., and Kay, J . (1990). Sensitive, soluble chromogenic substrates for HIV-1 proteinase. J. Biol. Chem. $265,7733-7736$.

Schramm, H.J ., Nakashima, H., Schramm, W., Wakayama, H., and Yamamoto, N. (1991). HIV-1 replication is inhibited by peptides derived from the $\mathrm{N}$ - and $\mathrm{C}$-termini of HIV-1 protease. Biochem. Biophys. Res. Commun. 179, 847-851.

Schramm, H.J ., Boetzel, J ., Büttner, J ., Fritsche, E., Göhring, W., J aeger, E., König, S., Thumfart, O., Wenger, T., Nagel, N.E., and Schramm, W. (1996). Inhibition of human immuno-deficiency virus proteases by 'interface peptides'. Antiviral Res. 30, 155 170.

Schulze, A.J ., Frohnert, P.W., Engh, R.A., and Huber, R. (1992). Evidence for the extent of insertion of the active site loop of intact $\alpha 1$ proteinase inhibitor in $\beta$-sheet A. Biochemistry 31, $7560-7565$

Uhlíková, T., Konvalinka, J ., Pichová, I., Soucek, M., Kräusslich, H.-G., Vondrášek, J . (1996). A modular approach to HIV-1 proteinase inhibitor design. Biophys. Biochem. Res. Commun. $222,38-43$.

Wlodawer, A., and Vondrasek, J . (1998). Inhibitors of HIV-1 protease: A major success of structure-assisted drug design. Ann. Rev. Biophys. Biomo. Struct., 27, 249- 284.

Zhang, Z.-Y., Poorman, R.A., Maggiora, L.L., Heinrikson, R.L., and Kezdy, F.J . (1991). Dissociation inhibition of dimeric enzymes. Kinetic characterisation of the inhibition of HIV-1 protease by its $\mathrm{COOH}$-terminal tetrapeptide. J. Biol. Chem. 266, $15591-155$

Zutshi, R., Franciskovich, J ., Shultz, M., Schweitzer, B., Bishop, P., Wilson, M., and Chmielewski, J . (1997). Targeting the dimerization interface of HIV-1 protease: inhibition with crosslinked interfacial peptides. J. Amer. Chem. Soc. 119, 4841- 4845.

Zutshi, R., B rickner, M., and Chmielewski, J . (1998). Inhibiting the assembly of protein-protein interfaces. Curr. Opin. Chem. Biol. $2,62-66$.

Received December 22,1998; accepted March 10,1999 